\title{
A higher dose of vancomycin is needed in critically ill patients with augmented renal clearance
}

\author{
Juan $\mathrm{He}^{1}$, Zhi-Tao Yang ${ }^{2}$, Xian Qian ${ }^{3}$, Bing Zhao ${ }^{2}$, En-Qiang $\mathrm{Mao}^{2}$, Er-Zhen Chen ${ }^{2}$, Xiao-Lan Bian ${ }^{1}$ \\ ${ }^{1}$ Department of Pharmacy, Ruijin Hospital Affiliated to Shanghai Jiao Tong University School of Medicine, Shanghai, China; ${ }^{2}$ Department of \\ Emergency Intensive Care Unit, Ruijin Hospital Affiliated to Shanghai Jiao Tong University School of Medicine, Shanghai, China; ${ }^{3}$ Department of \\ Pharmacy, Shanghai Eastern Hepatobiliary Surgery Hospital, Shanghai, China \\ Contributions: (I) Conception and design: J He, ZT Yang; (II) Administrative support: EQ Mao, EZ Chen, XL Bian; (III) Provision of study materials \\ or patients: B Zhao, EQ Mao, EZ Chen; (IV) Collection and assembly of data: J He, ZT Yang, X Qian; (V) Data analysis and interpretation: J He, \\ ZT Yang, X Qian, B Zhao; (VI) Manuscript writing: All authors; (VII) Final approval of manuscript: All authors. \\ Correspondence to: Xiao-Lan Bian. Department of Pharmacy of Ruijin Hospital Affiliated to Shanghai Jiao Tong University School of Medicine, \\ 202, No. 12 building, 197 Ruijin, No. 2 Road, Huangpu District, Shanghai, China. Email: bxl70029@163.com. En-Qiang Mao; Erzhen Chen. \\ Department of Emergency Intensive Care Unit, Ruijin Hospital Affiliated to Shanghai Jiao Tong University School of Medicine, EICU 3 floor, 5 \\ building, 197 Ruijin No. 2 Road, Huangpu District, Shanghai, China. Email: maoeq@yeah.net; chenerzhen@hotmail.com.
}

Background: Using standard vancomycin dosage in critically ill patients might lead to therapy failure and worse patient outcomes, augmented renal clearance (ARC) may be the leading risk factor. In this study, we comprehensively investigated the pharmacokinetics-pharmacodynamics (PK-PD) of vancomycin in critically ill patients with ARC, hoping to explore the precise and accurate dose adjustment method for vancomycin.

Methods: All critically ill patients tested for steady-state trough vancomycin serum concentrations during the recent 6 years in a tertiary level hospital were collected retrospectively and divided into ARC and nonARC groups, respectively, according to creatinine clearance (CLcr). Serum vancomycin concentrations were measured by the fluorescence polarization immunoassay method. PK-PD parameters of vancomycin were recorded or calculated. The desired daily dose successful in achieving the lower target trough levels $(10 \mathrm{mg} / \mathrm{L})$ of vancomycin were investigated correspondingly.

Results: A total of 280 vancomycin concentrations were eligible for analysis. The ARC group $(\mathrm{n}=139)$ contained more male patients $(64.7 \%)$ with average age and CLcr of 40 years old $(\mathrm{P}<0.05)$ and $180.8 \mathrm{~mL} / \mathrm{min}$ $(\mathrm{P}<0.001)$, respectively. Those patients exhibited higher clearance $(\mathrm{CL})$ and lower trough serum concentrations than the non-ARC patients under comparable daily doses of vancomycin. All the ICU patients demonstrated lower $\mathrm{AUC}_{24 \mathrm{~h}}$ values than the target level of $400 \mu \mathrm{g} \cdot \mathrm{h} / \mathrm{mL}$, and this value showed a lower trend in the ARC group than the non-ARC group (232.9 vs. $316.2 \mu \mathrm{g} \cdot \mathrm{h} / \mathrm{mL}$ ). Subtherapeutic trough concentrations of vancomycin $(<10.0 \mathrm{mg} / \mathrm{L})$ were observed in $77.7 \%$ and $68.8 \%$ of the ARC and non-ARC patients $(\mathrm{P}<0.05)$. The proportion of patients with a trough concentration of $10-15$ and $15-20 \mathrm{mg} / \mathrm{L}$ was $17.9 \%$ and $4.3 \%$, respectively, in the ARC group and $24.8 \%$ and $2.8 \%$, respectively, in the non-ARC group., a daily dose of 46.0 and $35.5 \mathrm{mg} / \mathrm{kg}$ of vancomycin is needed, respectively, in the ARC and non-ARC group to achieve a target trough concentration of $10 \mathrm{mg} / \mathrm{L}$.

Conclusions: A higher dose of vancomycin is needed in critically ill patients, especially those with ARC, and appropriate TDM-guided dose adjustment should be considered to achieve the targeted therapeutic range and to provide dosing guidance for this: patient population.

Keywords: Augmented renal clearance (ARC); critically ill; vancomycin; PK-PD

Submitted Jun 03, 2020. Accepted for publication Sep 16, 2020.

doi: $10.21037 /$ tau-20-1048

View this article at: http://dx.doi.org/10.21037/tau-20-1048 


\section{Introduction}

Adequate antibiotic therapy is of pivotal importance in the treatment of infections in critically ill patients. However, the pharmacokinetics of those ill patients can be significantly altered due to augmented renal clearance (ARC) of drugs eliminated via kidneys (1). ARC has been reported in 30 $65 \%$ of patients in the intensive care unit (ICU) (2). There is a clear association between ARC and subtherapeutic antibiotic concentrations, as well as literature suggesting worse clinical outcomes $(3,4)$.

Currently, vancomycin is still suggested as the firstline treatment against infections with Gram-positive bacteria, especially for the treatment of methicillinresistant Staphylococcus aureus (MRSA) infections. To achieve therapeutic concentrations and avoid nephrotoxicity, the Chinese guidelines recommend that target trough serum concentrations of $10-20 \mathrm{mg} / \mathrm{L}$ are required to achieve an area-under-concentration-time-curve (AUC) to minimum-inhibitory-concentration (MIC) ratio (AUC/ MIC) of $>400$. As a result, optimizing of vancomycin therapy by therapeutic drug monitoring (TDM) is widely recommended (5). However, in the clinical practice, our previous study revealed that the serum trough concentration of vancomycin was even much lower than $10-20 \mathrm{mg} / \mathrm{L}$ when infused with the suggested dose from drug instructions or guidelines in patients with severe acute pancreatitis, and ARC may be the main risk factor (6). Since vancomycin is water soluble and can be cleared predominantly by kidney, infusion with an empirical dosage in patients with ARC would potentially result in failed treatment with low drug exposure or bacterial resistance (7). The present study was to provide pharmacokinetics-pharmacodynamics (PK-PD) parameters of serum vancomycin and to analyze the optimal dosage regimen in critically ill patients with ARC, hoping to explore precise and accurate dose adjustment methods for vancomycin. To our best knowledge, this is the first study suggesting the optimal dosage regimen of vancomycin in critically ill patients with ARC in the Chinese population.

We present the following article in accordance with the STROBE reporting checklist (available at http://dx.doi. org/10.21037/tau-20-1048).

\section{Methods}

\section{Subjects}

All critically ill patients tested for steady-state trough vancomycin serum concentrations during the last 6 years
(January 2013-November 2018) in which a tertiary level hospital was analyzed. The study was approved by the local Ethics Committee of Ruijin Hospital, who waived the need for informed consent in consideration of the retrospective and observational nature of the study, and the study was performed following the ethical standards laid down in the Declaration of Helsinki (as revised in 2013). Patients with poor renal function (eCLcr $<80 \mathrm{~mL} / \mathrm{min}$ ), pregnant or lactating are excluded. Two hundred eighty vancomycin concentrations were eligible for analysis: 139 (50\%) and 141 cases were assigned to the ARC (eCLcr, creatinine clearance, estimated by Cockcroft-Gault, $>130 \mathrm{~mL} / \mathrm{min}$ ) and the non-ARC groups (eCLcr in the range, $80-130 \mathrm{~mL} / \mathrm{min}$ ), respectively.

\section{Vancomycin TDM and Pharmacokinetic analysis}

Fifteen $\mathrm{mg} / \mathrm{kg}$ initial dosage of vancomycin was administered intravenously every $12 \mathrm{~h}$ lasted for $2 \mathrm{hrs}$ in the critically ill patients. Serum vancomycin concentrations were measured by the fluorescence polarization immunoassay method using an Integra700 or a Cobas6000 c501 analyzer (Roche Diagnostics, Shanghai, China). The lower and upper limits of quantification of the vancomycin assay were 0 and $100.0 \mathrm{mg} / \mathrm{L}$, respectively. Pharmacokinetic parameters of vancomycin are estimated using the Bayesian estimator JPKD Ver. 3.1, which was developed with the population pharmacokinetic analysis with a twocompartment model in a Spanish population (8). $\mathrm{AUC}_{24 \mathrm{~h}}$ was calculated using the trapezoidal area method through the steady-state serial concentrations of $0,1,2,4,6,8,12$, and $24 \mathrm{~h}$ before and after vancomycin administration, which was estimated via the above mentioned Bayesian estimator. The MIC of the Gram-positive bacteria (including Staph Aureus, Enterococcus faecalis, and Enterococcus faecium) were recorded, the $\mathrm{AUC}_{24 \mathrm{~h}} / \mathrm{MIC}$ was calculated using the average MIC for each group, respectively. Vancomycin TDM has been implemented, and dose adjustment was with the trough level 5 days post vancomycin initiation. The desired daily dose successful in achieving the target trough levels $(10 \mathrm{mg} / \mathrm{L})$ of vancomycin was recorded using the Bayesian estimator JPKD Ver. 3.1.

\section{Statistical analysis}

Numerical variables are summarized as the mean \pm standard deviation (SD). Parametric and nonparametric analyses were performed using SPSS statistical software (version 21.0, SPSS 
Inc., Chicago). Determination of the significant differences in parameters between both groups was performed by either the Student's $t$-test or the Mann-Whitney U test. A P value of $<0.05$ was statistically significant.

\section{Results}

\section{The characteristics and the main pathogenies of the patients}

The characteristics and the main pathogenies are summarized in Table 1 . The ARC group contains more male patients (64.7\%) with average age and CLcr of 40 years old $(\mathrm{P}<0.05)$ and $180.8 \mathrm{~mL} / \mathrm{min}(\mathrm{P}<0.001)$, respectively. The average daily dose of vancomycin was $15.0 \mathrm{mg} / \mathrm{kg}$ (q12h) and comparable among the groups. The patients with ARC exhibited higher vancomycin clearance (CL) and lower trough serum vancomycin concentration values than nonARC patients.

\section{The PK-PD parameters of the patients}

All the ICU patients demonstrated lower $\mathrm{AUC}_{24 \mathrm{~h}}$ values than the target level of $400 \mu \mathrm{g} \cdot \mathrm{h} / \mathrm{mL}$ recommended for $S$. aureus, and this value showed a lower trend in the ARC group than the non-ARC group (232.9 vs. $316 \mu \mathrm{g} \cdot \mathrm{h} / \mathrm{mL}$ ). There was no significant difference in the apparent volume of distribution $(\mathrm{Vd})$ for vancomycin between the patients with or without ARC (Table 1). The MIC values of the Gram-positive bacteria (Staph Aureus, Enterococcus faecalis, and Enterococcus faecium) were recorded, and the $\mathrm{AUC}_{24 \mathrm{~h}}$ / MIC was calculated using average MIC for each group, respectively (Table 1). Subtherapeutic trough concentrations of vancomycin $(<10.0 \mathrm{mg} / \mathrm{L})$ were observed in $77.7 \%$ of the ARC patients and $68.8 \%$ of the non-ARC patients $(\mathrm{P}<0.05)$. The proportion of patients with a trough concentration of $10-15$ and $15-20 \mathrm{mg} / \mathrm{L}$ was $17.9 \%$ and $4.3 \%$, respectively, in the ARC group and $24.8 \%$ and $2.8 \%$, respectively, in the non-ARC group. A daily dose of 46.0 and $35.5 \mathrm{mg} / \mathrm{kg}$ of vancomycin is needed, respectively, in the ARC and nonARC group to achieve a target trough concentration of 10 $\mathrm{mg} / \mathrm{L}$.

\section{Discussion}

ARC is a commonly occurring clinical phenomenon with worse clinical outcomes in critically ill patients receiving antimicrobial therapy (9). Those patients are often exposed to factors that may increase the likelihood of ARC occurrences, including infection, trauma, pancreatitis, burns, major surgery, and using intravenous fluids, vasopressors, and inotropes. Minkute et al. revealed new significant conditions to predict ARC: mechanical ventilation and hemodynamically unstable status, which are common in ICU (7). Udy et al. have reported that $65 \%$ of ICU patients with ARC, had at least 1 occasion of measuring ARC during the first 7 days of ICU admission (10). The present study focused on ICU patients with 'normal' renal function, and the results showed that ARC could be found in up to half of the patients with an average CLcr of $180.8 \mathrm{~mL} / \mathrm{min}$ in this cohort of investigative patients. As vancomycin is suggested as the first-line treatment against infections with Gram-positive bacteria in these critically ill cases and subtherapeutic serum concentrations of vancomycin dominated in ICU patients, especially those with ARC in our study, this finding should be considered while prescribing vancomycin.

ARC was more relevant in younger male patients (7), and results of our study confirmed this statement: most patients with ARC are males (64.7\%) with an average age of 40 years old while there were $53.2 \%$ males with an average age of 55 years old $(\mathrm{P}<0.05)$ in the non-ARC group in our study. Results in body weight and Apache II score were comparable without significant difference in both groups. However, higher CLcr and lower serum concentrations of vancomycin were more often detected in the ARC group $(\mathrm{P}<0.001$ and $\mathrm{P}<0.01$, respectively) under a comparable daily dose of $30 \mathrm{mg} / \mathrm{kg} / \mathrm{d}$. The usual dosage of the drug showed lower than $30 \%$ of therapeutic trough concentrations of vancomycin in both ARC (77.8\% subtherapeutic and 22.2\% therapeutic) and the control groups $(68.8 \%$ subtherapeutic and $27.7 \%$ therapeutic). Comparable results were revealed by studies of Ocampos-Martinez et al., who reported higher proportions of insufficient vancomycin concentrations in patients with similar CLcr (11). Enhanced vancomycin clearance will lead to shorter drug half-life $\left(\mathrm{t}_{1 / 2}\right)$ and lower AUC, which could have a direct implication on drugs' pharmacodynamic effects. These PK-PD changes to drug therapy in ARC may have drastic implications on patient outcomes. Previous studies have established that steadystate vancomycin trough concentration is an adequate surrogate of AUC/MIC, which is, in turn, correlated with treatment success (12). An $\mathrm{AUC}_{24 \mathrm{~h}} / \mathrm{MIC}>350$ was associated with clinical success, and an $\mathrm{AUC}_{24 \mathrm{~h}} / \mathrm{MIC}>400$ indicated faster bacterial eradication in patients receiving vancomycin for Staphylococcus aureus pneumonia (13). In our 
Table 1 Characteristics, pathogenies and PK-PD parameters in the ARC and non-ARC group ( $\mathrm{n}=280)$

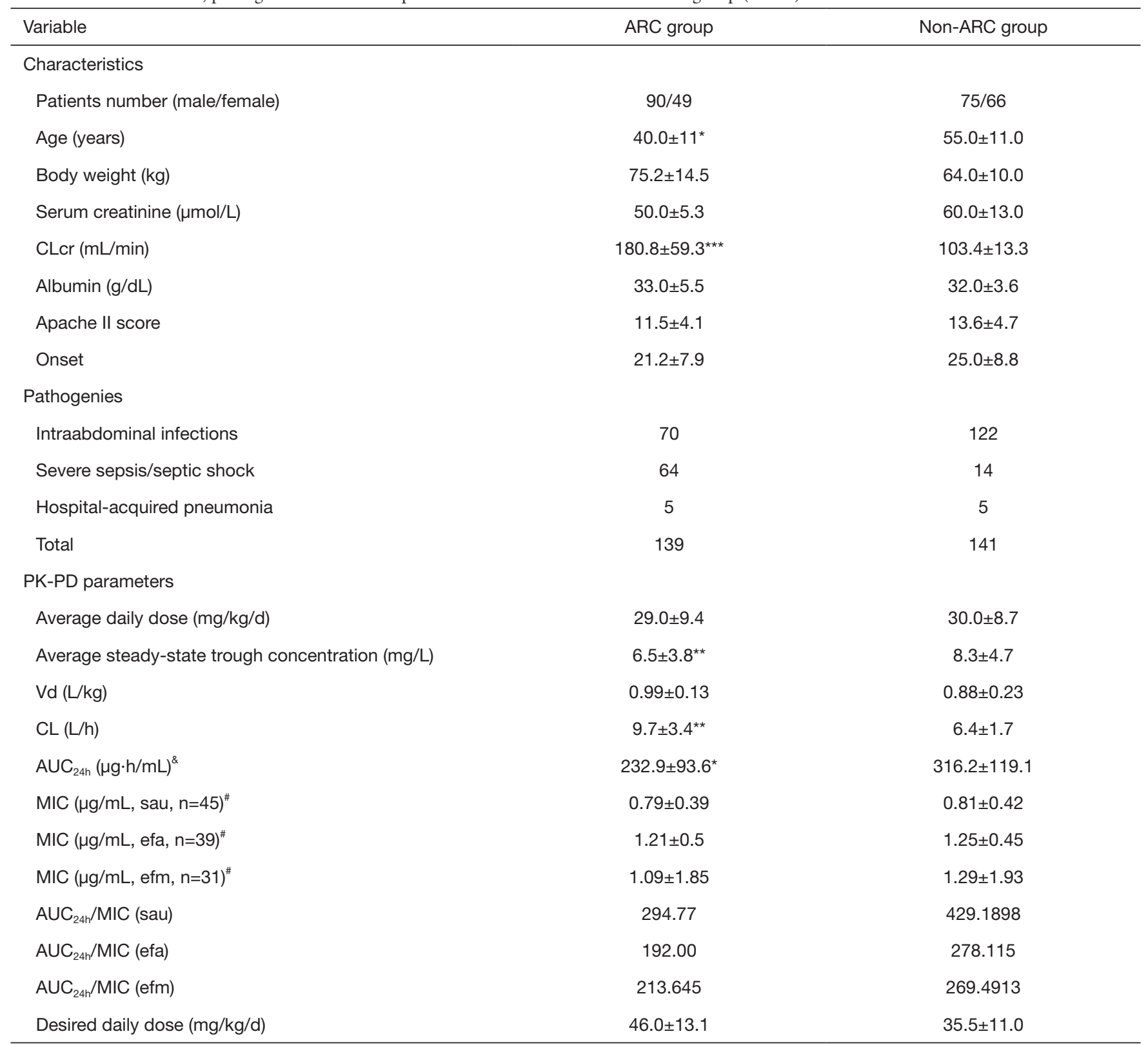

*, $\mathrm{P}<0.05$; ${ }^{* *}, \mathrm{P}<0.01$; ${ }^{* *}, \mathrm{P}<0.001$ (compared with non-ARC group); ${ }^{*}, \mathrm{AUC}_{24 \mathrm{~h}}$ was calculated using trapezoidal area method through the steady state serial concentrations of $0,1,2,4,6,8,12$ and $24 \mathrm{~h}$ before and after vancomycin administration. The concentrations were estimated using the Bayesian estimator JPKD Ver. 3.1. ", average MIC value of isolated Staphylococcus aureus (sau, $\mathrm{n}=20$ ), Enterococcus faecalis (efa, $\mathrm{n}=14$ ) and Enterococcus faecium (efm, $\mathrm{n}=10$ ) in each group.

present study, we revealed that all the ARC patients did not have $\mathrm{AUC}_{24 \mathrm{~h}} / \mathrm{MIC}$, levels above the target $350 \mu \mathrm{g} \cdot \mathrm{h} / \mathrm{mL}$, and even lower than $300 \mu \mathrm{g} \cdot \mathrm{h} / \mathrm{mL}$ under a conventional dosage regimen, the values were much lower in those who were infected with Enterococcus faecalis and Enterococcus faecium, due to higher MIC values of these pathogens.
Similar results were demonstrated by Carlier et al. who showed the target PK-PD was significantly lower in patients with ARC than those without ARC $\left(50 \% f \mathrm{~T}_{>M I C}\right.$ and $100 \% f \mathrm{~T}_{>\text {MIC }}$, respectively) within 61 patients who received meropenem ( $1 \mathrm{~g} \mathrm{q} 8 \mathrm{~h}$ ) or piperacillin/tazobactam (4.5 $\mathrm{g} \mathrm{q} 6 \mathrm{~h}$ ) as an extended infusion for $3 \mathrm{~h}$ (14). In patients who did not 
reach the target $15-25 \mathrm{mg} / \mathrm{L} 48 \mathrm{~h}$ post-treatment initiation, in-hospital mortality was significantly higher than those who have attained a therapeutic target trough $(\mathrm{OR}=2.1$, $\mathrm{P}=0.003)$ (14). These facts emphasize a general warning about the risk for underexposure in the case of critically ill should be warranted. They also highlight the need for dosing guidance, especially in those with ARC. In our present study, vancomycin TDM has been implemented, and dose adjustment was with the trough level 5 days post vancomycin initiation. A desired dose of 46.0 and $35.5 \mathrm{mg} / \mathrm{kg} / \mathrm{day}$ were needed in patients with or without $\mathrm{ARC}$, respectively, to reach the target trough levels of $10 \mathrm{mg} / \mathrm{L}$. In severe cases, to achieve the target of $15 \mathrm{mg} / \mathrm{L}$, our study suggested a loading dose of $25 \mathrm{mg} / \mathrm{kg}$ followed by a daily dose of 69 and $53 \mathrm{mg} / \mathrm{kg}$ for those with and without ARC, respectively. A vancomycin dosing algorithm could also be implemented to allow a rapid achievement of a target trough concentration by the estimation of ARC, which implies an adjustment of the dose of vancomycin, both in terms of loading and continuous infusion dose (15). Patients' pathophysiological state and TDM results should always be stressed to adjust the dosage for individual administration.

\section{Conclusions}

A higher dose of vancomycin is needed in critically ill patients, especially those with ARC, and appropriate TDMguided dose adjustment should be considered to achieve the targeted therapeutic range and to optimize the PK-PD properties. Further clinical studies are necessary to confirm these observations, which could help with the establishment of guidelines for vancomycin dosing strategy in ICU patients with ARC.

\section{Acknowledgments}

The authors thank the staff of the emergency ICU Department of Ruijin Hospital Affiliated to Shanghai Jiao Tong University School of Medicine, for their facilities and collaboration.

Funding: This study was supported by the National Natural Science Foundation of China (Grant No. 81772107 and 81571931).

\section{Footnote}

Reporting Checklist: The authors have completed the
STROBE reporting checklist. Available at http://dx.doi. org/10.21037/tau-20-1048

Data Sharing Statement: Available at http://dx.doi. org/10.21037/tau-20-1048

Conflicts of Interest: All authors have completed the ICMJE uniform disclosure form (available at http://dx.doi. org/10.21037/tau-20-1048). The authors have no conflicts of interest to declare.

Ethical Statement: The authors are accountable for all aspects of the work in ensuring that questions related to the accuracy or integrity of any part of the work are appropriately investigated and resolved. Ruijin Hospital Institutional Review Board approved this study and the study was performed following the ethical standards laid down in the Declaration of Helsinki (as revised in 2013). Two hundred eighty patients were enrolled in the study, and informed consent forms were not available due to the retrospective and observational nature of the study.

Open Access Statement: This is an Open Access article distributed in accordance with the Creative Commons Attribution-NonCommercial-NoDerivs 4.0 International License (CC BY-NC-ND 4.0), which permits the noncommercial replication and distribution of the article with the strict proviso that no changes or edits are made and the original work is properly cited (including links to both the formal publication through the relevant DOI and the license). See: https://creativecommons.org/licenses/by-nc-nd/4.0/.

\section{References}

1. Claus BO, Hoste EA, Colpaert K, et al. Augmented renal clearance is a common finding with worse clinical outcome in critically ill patients receiving antimicrobial therapy. J Crit Care 2013;28:695-700.

2. Hobbs AL, Shea KM, Roberts KM, et al. Implications of Augmented Renal Clearance on Drug Dosing in Critically Ill Patients: A Focus on Antibiotics. Pharmacotherapy 2015;35:1063-75.

3. Baptista JP, Sousa E, Martins PJ, et al. Augmented renal clearance in septic patients and implications for vancomycin optimisation. Int J Antimicrob Agents 2012;39:420-3.

4. Jeurissen A, Sluyts I, Rutsaert R. A higher dose of vancomycin in continuous infusion is needed in critically ill patients. Int J Antimicrob Agents 2011;37:75-7. 
5. Ishii H, Hirai K, Sugiyama K, et al. Validation of a Nomogram for Achieving Target Trough Concentration of Vancomycin: Accuracy in Patients With Augmented Renal Function. Ther Drug Monit 2018;40:693-8.

6. He J, Mao EQ, Feng J, et al. The pharmacokinetics of vancomycin in patients with severe acute pancreatitis. Eur J Clin Pharmacol. 2016;72:697-702.

7. Minkutè R, Briedis V, Steponavičiūtè R, et al. Augmented renal clearance--an evolving risk factor to consider during the treatment with vancomycin. J Clin Pharm Ther 2013;38:462-7.

8. Available online: http://pkpd.kmu.edu.tw/jpkd/ downloadJPKD.htm

9. Mahmoud SH, Shen C. Augmented Renal Clearance in Critical Illness: An Important Consideration in Drug Dosing. Pharmaceutics 2017;9:36.

10. Udy AA, Baptista JP, Lim NL, et al. Augmented renal clearance in the ICU: results of a multicenter observational study of renal function in critically ill patients with normal plasma creatinine concentrations*. Crit Care Med 2014;42:520-7.

11. Ocampos-Martinez E, Penaccini L, Scolletta S, et

Cite this article as: $\mathrm{He} \mathrm{J}$, Yang ZT, Qian X, Zhao B, Mao EQ, Chen EZ, Bian XL. A higher dose of vancomycin is needed in critically ill patients with augmented renal clearance. Transl Androl Urol 2020;9(5):2166-2171. doi:10.21037/tau-20-1048 al. Determinants of early inadequate vancomycin concentrations during continuous infusion in septic patients. Int J Antimicrob Agents 2012;39:332-7.

12. Rybak MJ. The pharmacokinetic and pharmacodynamic properties of vancomycin. Clin Infect Dis 2006;42 Suppl 1: S35-9.

13. Moise-Broder PA, Forrest A, Birmingham MC, et al. Pharmacodynamics of vancomycin and other antimicrobials in patients with Staphylococcus aureus lower respiratory tract infections. Clin Pharmacokinet 2004;43:925-42.

14. Carlier M, Carrette S, Roberts JA, et al. Meropenem and piperacillin/tazobactam prescribing in critically ill patients: does augmented renal clearance affect pharmacokinetic/ pharmacodynamic target attainment when extended infusions are used? Crit Care 2013;17:R84.

15. Spadaro S, Berselli A, Fogagnolo A, et al. Evaluation of a protocol for vancomycin administration in critically patients with and without kidney dysfunction. BMC Anesthesiol 2015;15:95.

(English Language Editor: J. Chapnick) 